\title{
Some Properties for Bisemivalues on Bicooperative Games
}

\author{
Margarita Domènech · José Miguel Giménez ·
}

María Albina Puente

Communicated by Kyriakos G. Vamvoudakis

Received: date / Accepted: date

\begin{abstract}
In this work, we focus on bicooperative games, a variation of the classic cooperative games, and investigate the conditions for the coefficients of the bisemivalues - a generalization of semivalues for cooperative games - necessary and / or sufficient in order to satisfy some properties, including among others, desirability
\end{abstract}

Margarita Domènech

Universitat Politècnica de Catalunya

Manresa, Spain

margarita.domenech@upc.edu

José Miguel Giménez

Universitat Politècnica de Catalunya

Manresa, Spain

jose.miguel.gimenez@upc.edu

María Albina Puente, Corresponding author

Universitat Politècnica de Catalunya

Manresa, Spain

m.albina.puente@upc.edu 
relation, balanced contributions, null player exclusion property and block property. Moreover, a computational procedure to calculate bisemivalues in terms of the multilinear extension of the game is given.

Keywords Bicooperative game $\cdot$ Bisemivalues $\cdot$ Regularity $\cdot$ Multilinear extension.

Mathematics Subject Classification (2000) $91 \mathrm{~A} 12$

\section{Introduction}

Cooperative games in a finite set of players are often defined in terms of a characteristic function, which specifies the worth that each coalition can achieve for itself independently of the remaining players. Each player has only two options: either to join a coalition (in this case, he or she is supposed to cooperate in order to obtain the maximum worth of the coalition) or to stay aside. However, there are many situations, that cannot be described by using this classical model, that we will see in the final section of the paper. Introduced in [1], bicooperative games are appropriate in this kind of scenarios.

In these games, each player can participate positively to the game, negatively, or do not participate. Formally, ordered pairs of disjoint coalitions of players have to be considered. Thus, each such pair yields a partition of the set of players in three groups: (i) players in the first coalition are defenders of modifying the actual situation and they want to accept a proposal; (ii) players in the second coalition do not agree with it and they will take actions against any change; and (iii) the remaining players are not convinced of the profits of the change, but they do not have intention of objecting to it. 
As in the cooperative case, different solution concepts have been introduced on bicooperative games. In [2], the Shapley value for bicooperative games is introduced and in [3], biprobabilistic values for bicooperative games were defined and characterized. In [4], ternary bicooperative games, which are a refinement of the ternary voting games introduced in [5], were analized and the Banzhaf power index was defined and axiomatized for these games.

In a recent paper [6], bisemivalues on bicooperative games were introduced and an interesting characterization of this kind of values by means of weighting coefficients was provided, in a similar way as given for semivalues on cooperative games. Moreover, a subfamily of these values, called $(p, q)$-bisemivalues was also introduced. The multilinear extension of a bicooperative game was also defined and, for the particular case of the $(p, q)$-bisemivalues, a computational procedure in terms of the multilinear extension of the game to calculate them, was also given.

The first part of the present paper focusses on studying the behavior of the bisemivalues with respect to several standard properties considered for values on cooperative games, concerning dominance, monotonicity, sensitivity, null and nonnull players, balanced contributions and block (see, e.g., $[5,7,8]$ ). The notions of regularity and induced bisemivalue arise in a natural way as a convenient condition to guarantee the validity of some of them. We then combine the notion of induced bisemivalue on lower cardinalities with regularity and obtain a series of characteristic properties of regular bisemivalues that concerns null and nonnull players, subgames and blocks.

The second part is devoted to give a computational procedure to compute bisemivalues by means of the multilinear extension of the game, that parallels the method 
obtained in [9] to compute semivalues on cooperative games and expands the results obtained in [6] for $(p, q)$-bisemivalues.

The organization of the paper is as follows. In Section 2, a minimum of preliminaries is provided. In Section 3, we introduce several properties for values on bicooperative games and study the behavior of bisemivalues with respect to them. Section 4 is devoted to give a procedure to compute bisemivalues. Section 5 contains one example and finally, Section 6 concludes.

\section{Preliminaries}

\subsection{Cooperative Games and Semivalues}

Let $N$ be a finite set of players and $2^{N}$ be the set of its coalitions (subsets of $N$ ). A cooperative game on $N$ is a function $v: 2^{N} \rightarrow \mathbb{R}$, that assigns a real number $v(S)$ to each coalition $S \subseteq N$, with $v(\emptyset)=0$. We will denote by $\mathcal{G}_{N}$ the set of all cooperative games on $N$.

Following the axiomatic description given in [10], $\psi: \mathcal{G}_{N} \rightarrow \mathbb{R}^{N}$ is a semivalue iff it satisfies the following properties: (i) linearity: $\psi\left[v+v^{\prime}\right]=\psi[v]+\psi\left[v^{\prime}\right]$ and $\psi[\lambda v]=$ $\lambda \psi[v]$ for all $v, v^{\prime} \in \mathcal{G}_{N}$ and $\lambda \in \mathbb{R}$; (ii) anonymity: $\psi_{\theta i}[\theta v]=\psi_{i}[v]$ for all permutations $\theta$ on $N, i \in N$, and $v \in \mathcal{G}_{N}$; (iii) positivity: if $v$ is monotonic, then $\psi[v] \geq 0$ and (iv) dummy player property: if $i \in N$ is a dummy in game $v$, then $\psi_{i}[v]=v(\{i\})$.

In [11], there is an interesting characterization of semivalues by means of weighting coefficients. Set $n=|N|$. Then: (a) for every weighting vector $\left\{p_{k}\right\}_{k=0}^{n-1}$ such that 
$\sum_{k=0}^{n-1} p_{k}\left(\begin{array}{c}n-1 \\ k\end{array}\right)=1$ and $p_{k} \geq 0$ for all $k$, the expression

$$
\psi_{i}[v]=\sum_{S \subseteq N \backslash\{i\}} p_{S}[v(S \cup\{i\})-v(S)] \quad \text { for all } i \in N \text { and all } v \in \mathcal{G}_{N}
$$

where $s=|S|$, defines a semivalue $\psi$; (b) conversely, every semivalue can be obtained in this way; (c) the correspondence given by $\left\{p_{k}\right\}_{k=0}^{n-1} \mapsto \psi$ is bijective.

Thus, the payoff that a semivalue allocates to every player in any game is a weighted sum of his marginal contributions in the game. If $p_{k}$ is interpreted as the probability that a given player $i$ joins a coalition of size $k$, provided that all the coalitions of a common size have the same probability of being joined, then $\psi_{i}[v]$ is the expected marginal contribution of that player to a random coalition he joins.

Well known examples of semivalues are the Shapley value $\varphi,[12]$, for which $p_{k}=1 / n\left(\begin{array}{c}n-1 \\ k\end{array}\right)$, and the Banzhaf value $\beta$ [13], for which $p_{k}=2^{1-n}$. The Shapley value is the only efficient semivalue, in the sense that $\sum_{i \in N} \varphi_{i}[v]=v(N)$ for every $v \in \mathcal{G}_{N}$.

The multilinear extension [14] of a game $v \in \mathcal{G}_{N}$ is the real-valued function defined on $\mathbb{R}^{N}$ by

$$
f_{v}\left(X_{N}\right)=\sum_{S \subseteq N} \prod_{i \in S} x_{i} \prod_{j \in N \backslash S}\left(1-x_{j}\right) v(S)
$$

where $X_{N}$ denotes the set of variables $x_{i}$ for $i \in N$ and $0 \leq x_{i} \leq 1$ for $i=1,2, \ldots, n$.

\subsection{Bicooperative Games and Bisemivalues}

Let $N$ be a finite set of players and $3^{N}=\{(S, T): S, T \subseteq N, S \cap T=\emptyset\}$ be the set of all ordered pairs of disjoint coalitions. Following [15], there is a relation in $3^{N}$ given by $(A, B) \sqsubseteq(C, D) \Leftrightarrow A \subseteq C, B \supseteq D$. 
Following [1], a bicooperative game on $N$ is a function $b: 3^{N} \rightarrow \mathbb{R}$, that assigns a real number $b(S, T)$ to each pair of coalitions $(S, T) \in 3^{N}$, with $b(\emptyset, \emptyset)=0$. For each $(S, T) \in 3^{N}$, the worth $b(S, T)$ represents the maximal gain (if $b(S, T)>0$ ) or the minimal loss (if $b(S, T)<0$ ) that is obtained, when players in $S$ are in favor of a change in the situation, players in $T$ are against the change and players in $N \backslash(S \cup T)$ are indifferent. Then, $b(\emptyset, N)$ is the cost obtained, when all players are against the change and $b(N, \emptyset)$ is the maximal gain obtained, when all players want to change the initial situation. We will denote by $\mathcal{B} \mathcal{G}_{N}$ the set of all bicooperative games on $N$.

A bicooperative game is monotonic if $b(S, T) \leq b\left(S^{\prime}, T^{\prime}\right)$, whenever $(S, T) \sqsubseteq$ $\left(S^{\prime}, T^{\prime}\right)$. A player $i \in N$ is a dummy in $b$ if $b(S \cup\{i\}, T)=b(S, T)+b(\{i\}, \emptyset)$ and $b\left(S, T \cup\{i\}=b(S, T)+b(\emptyset,\{i\})\right.$ for all $(S, T) \in 3^{N \backslash\{i\}}$, and null in $b$ if, moreover, $b(\{i\}, \emptyset)=b(\emptyset,\{i\})=0$.

Endowed with the natural operations for real-valued functions, the set of all bicooperative games on $N$ is a vector space $\mathcal{B} \mathcal{G}_{N}$. For every $(S, T) \in 3^{N}$ such that $(S, T) \neq$ $(\emptyset, \emptyset)$, the identity game $\delta_{(S, T)}$ is defined by $\delta_{(S, T)}(A, B)=1$, if $(A, B)=(S, T)$ and $\delta_{(S, T)}(A, B)=0$, otherwise. It is easily checked that the set of all identity games is a basis for $\mathcal{B} \mathcal{G}_{N}$, so that $\operatorname{dim}\left(\mathcal{B} \mathcal{G}_{N}\right)=3^{n}-1$ if $n=|N|$.

By a value on $\mathcal{B} \mathcal{G}_{N}$ we will mean a map $g: \mathcal{B} \mathcal{G}_{N} \rightarrow \mathbb{R}^{N}$, that assigns to every game $b$ a vector $g[b]$ with components $g_{i}[b]$ for all $i \in N$.

Bisemivalues for bicooperative games were defined and characterized in [6] as follows.

Definition 2.1 (Domènech et al, 2018) A bisemivalue on $\mathcal{B} \mathcal{G}_{N}$ is a map $\psi: \mathcal{B} \mathcal{G}_{N} \rightarrow \mathbb{R}^{N}$ that satisfies: 
(i) linearity: $\psi\left[\alpha b+\beta b^{\prime}\right]=\alpha \psi[b]+\beta \psi\left[b^{\prime}\right]$, for all $b, b^{\prime} \in \mathcal{G} \mathcal{B}_{N}$ and $\alpha, \beta \in \mathbb{R}$;

(ii) anonymity: $\psi_{\pi i}[\pi b]=\psi_{i}[b]$ for all permutation $\pi$ over $N, i \in N$, and $b \in \mathcal{B} \mathcal{G}_{N}$, where $\pi b(\pi S, \pi T)=b(S, T)$ and $\pi S=\{\pi i: i \in S\}$;

(iii) positivity: if $b$ is monotonic, then $\psi[b] \geq 0$;

(iv) dummy player property: if $i \in N$ is a dummy in game $b$ then, $\psi_{i}[b]=b(\{i\}, \emptyset)-$ $b(\emptyset,\{i\})$.

Theorem 2.1 (Domènech et al, 2018) A value $\psi$ on $\mathcal{B G}_{N}$ is a bisemivalue if and only if there exist two collections of real numbers $p_{s, t}$ and $q_{s, t}, s, t=0, .1, \ldots, n-1$, satisfying:

$$
\begin{aligned}
& p_{s, t} \geq 0, q_{s, t} \geq 0 \\
& \sum_{s=0}^{n-1}\left(\begin{array}{c}
n-1 \\
s
\end{array}\right)\left[\sum_{t=0}^{n-s-1}\left(\begin{array}{c}
n-s-1 \\
t
\end{array}\right) p_{s, t}\right]=1, \\
& \sum_{t=0}^{n-1}\left(\begin{array}{c}
n-1 \\
t
\end{array}\right)\left[\sum_{s=0}^{n-t-1}\left(\begin{array}{c}
n-t-1 \\
s
\end{array}\right) q_{s, t}\right]=1,
\end{aligned}
$$

such that

$$
\psi_{i}[b]=\sum_{(S, T) \in 3^{N \backslash i}}\left[p_{s, t}(b(S \cup i, T)-b(S, T))+q_{s, t}(b(S, T)-b(S, T \cup i))\right]
$$

for all $i \in N$ and all $b \in \mathcal{B} \mathcal{G}_{N}$, where $s=|S|$ and $t=|T|$.

In addition, a subfamily of bisemivalues, called $(p, q)$-bisemivalues, is introduced. For each one of them, the weighting coefficients depend on two parameters $p, q \in[0,1]$. These bisemivalues are suited for the study of bicooperative games where players show two different tendencies to form coalitions. These tendencies are defined for all players by parameters $p$ and $q$. 
Definition 2.2 (Domènech et al, 2018) Let $p, q \in[0,1]$ with $p+q \leq 1$. The $(p, q)$-bisemivalue $\psi^{p q}$ on $\mathcal{B} \mathcal{G}_{N}$ is defined by the coefficients $p_{s, t}=p^{s} q^{t}(1-p-q)^{n-s-t-1}$ and $q_{s, t}=p^{t} q^{s}(1-p-q)^{n-s-t-1}$.

Moreover, a subfamily of $(p, q)$-bisemivalues, obtained when $p=q$, and called binomial bisemivalues is also defined. They "extend" the concept of binomial semivalues, introduced in [16], to bicooperative games. Of course, $p=q=1 / 3$ gives the Banzhaf bisemivalue.

\section{Regularity and Other Properties}

In this section, we study for bisemivalues a series of standard properties considered in the literature on value theory. Most of them hold for semivalues on cooperative games but, as we will see, things are not so simple when we focus on bicooperative games and use bisemivalues. Some of these properties only hold for a special subclass of bisemivalues that we will call regular.

\subsection{Dominance, Monotonicity and Sensitivity Properties}

The first property concerns the desirability and indifference relations introduced in

[17] for cooperative games as follows. Let us considerer $v \in \mathcal{G}_{N}$ and $i, j \in N$. We say $i D j$ in $v$ iff $v(S \cup\{i\}) \geq v(S \cup\{j\})$ for all $S \subseteq N \backslash\{i, j\}$.

First of all, in the following definition we readapt this relation to bicooperative games in order to get the same interpretation as in the cooperative case. 
Definition 3.1 Let us considerer $b \in \mathcal{B} \mathcal{G}_{N}$ and $i, j \in N$.

$$
i D j \quad \text { in } b \text { iff }\left\{\begin{array}{l}
b(S \cup\{i\}, T) \geq b(S \cup\{j\}, T), \\
b(S, T \cup\{i\}) \leq b(S, T \cup\{j\}), \\
b(S \cup\{i\}, T \cup\{j\}) \geq b(S \cup\{j\}, T \cup\{i\}),
\end{array}\right.
$$

for all $(S, T) \in 3^{N \backslash\{i, j\}}$.

Relation $D$ allows us to qualitatively compare the positions of two players in a given game $b$.

Definition 3.2 Let us considerer $b \in \mathcal{B} \mathcal{G}_{N}$ and $i, j \in N$. $i I j$ in $b$ iff $i D j$ and $j D i$ in $b$.

Thus, $i D j$ in $b$ means that player $i$ dominates (i.e., is "at least as desirable as") $j$ as a coalition partner in $b$. Moreover, $i I j$ in $b$ means that players $i, j$ are symmetric, that is, indifferent (perfect substitutes of each other) as coalition partners. If $i D j$ but $j D i$ in $b$ then, we say that $i$ dominates $j$ strictly. It is not difficult to verify that $I$ is an equivalence relation (on $N)$.

As it is shown in [18], $g$ being a semivalue on $\mathcal{G}_{N}, i D j$ in $v$ implies $g_{i}[v] \geq g_{j}[v]$, and hence, $i I j$ in $v$ implies $g_{i}[v]=g_{j}[v]$, although not always $i D j$ and $j D^{\prime} i$ together in $v$ imply $g_{i}[v]>g_{j}[v]$. What happens when bicooperative games and bisemivalues are used?

Proposition 3.1 (Dominance property) Let $i, j \in N$ be two distinct players and let $\psi$ be a bisemivalue on $\mathcal{B} \mathcal{G}_{N}$. Then, for every game $b \in \mathcal{B G}_{N}$,

(a) iDj implies $\psi_{i}[b] \geq \psi_{j}[b]$ iff $p_{s, t}+p_{s+1, t} \geq q_{s+1, t}$ and $q_{s, t}+q_{s, t+1} \geq p_{s, t+1}$, for all $s, t=0, \ldots, n-2$. 
(b) $i D j$ and $j \not D i$ implies $\psi_{i}[b]>\psi_{j}[b]$ iff $p_{s, t}+p_{s+1, t}>q_{s+1, t}, p_{s, t+1}+q_{s+1, t}>0$ and $q_{s, t}+q_{s, t+1}>p_{s, t+1}$, for all $s, t=0, \ldots, n-2$.

Proof (a) $(\Leftarrow)$ Assume $i D j$ in $b$. Starting at Eq. (3) and splitting the sum into three parts, we have

$$
\begin{aligned}
\psi_{i}[b]= & \sum_{(S, T) \in 3^{N \backslash\{i\}}} p_{s, t}[b(S \cup i, T)-b(S, T)]+q_{s, t}[b(S, T)-b(S, T \cup i)]= \\
& \sum_{(S, T) \in 3^{\mathcal{Y} \backslash\{i, j\}}} p_{s, t}[b(S \cup i, T)-b(S, T)]+q_{s, t}[b(S, T)-b(S, T \cup i)]+ \\
& p_{s+1, t}[b(S \cup j \cup i, T)-b(S \cup j, T)]+q_{s+1, t}[b(S \cup j, T)-b(S \cup j, T \cup i)]+ \\
& p_{s, t+1}[b(S \cup i, T \cup j)-b(S, T \cup j)]+q_{s, t+1}[b(S, T \cup j)-b(S, T \cup i \cup j)] .
\end{aligned}
$$

Now, by comparing this expression with the analogous expression for $\psi_{j}[b]$, it follows that

$$
\begin{aligned}
\psi_{i}[b]-\psi_{j}[b]= & \sum_{(S, T) \in 3^{N \backslash\{i, j\}}}\left(p_{s, t}+p_{s+1, t}-q_{s+1, t}\right)[b(S \cup i, T)-b(S \cup j, T)]+ \\
& \left(q_{s, t}+q_{s, t+1}-p_{s, t+1}\right)[b(S, T \cup j)-b(S, T \cup i)]+ \\
& \left(p_{s, t+1}+q_{s+1, t}\right)[b(S \cup i, T \cup j)-b(S \cup j, T \cup i)] \geq 0 .
\end{aligned}
$$

$(\Rightarrow)$ (i) First, let us assume that $p_{a, b}+p_{a+1, b}<q_{a+1, b}$, for some $a, b=0, \ldots, n-2$. Pick $i, j \in N$ and $(A, B) \in 3^{N \backslash\{i, j\}}$ such that $|A|=a$ and $|B|=b$. Considerer the identity game $\delta_{(A \cup i, B)}$. It is clear that $i D j$ in this game however, $\psi_{i}\left[\boldsymbol{\delta}_{(A \cup i, B)}\right]-\psi_{j}\left[\boldsymbol{\delta}_{(A \cup i, B)}\right]=$ $p_{a, b}+p_{a+1, b}-q_{a+1, b}<0$, a contradiction.

(ii) Now, we assume that $q_{a, b}+q_{a, b+1}<p_{a, b+1}$, for some $a, b=0, \ldots, n-2$. Pick $i, j \in N$ and $(A, B) \in 3^{N \backslash\{i, j\}}$ such that $|A|=a$ and $|B|=b$. Considerer the identity game $\delta_{(A, B \cup j)}$. It is clear that $i D j$ in this game however, $\psi_{i}\left[\boldsymbol{\delta}_{(A, B \cup j)}\right]-\psi_{j}\left[\boldsymbol{\delta}_{(A, B \cup j)}\right]=$ $q_{a, b}+q_{a, b+1}-p_{a, b+1}<0$, a contradiction. 
(b) $(\Leftarrow)$ If $i D j$ and $j \not D i$ in b then, for some $(S, T) \in 3^{N \backslash\{i, j\}}$, we have either

$$
\begin{aligned}
& b(S \cup\{i\}, T)>b(S \cup\{j\}, T) \quad \text { or } \quad b(S, T \cup\{i\})<b(S, T \cup\{j\}) \quad \text { or } \\
& b(S \cup\{i\}, T \cup\{j\})>b(S \cup\{j\}, T \cup\{i\}) .
\end{aligned}
$$

As $p_{s, t}+p_{s+1, t}>q_{s+1, t}, p_{s, t+1}+q_{s+1, t}>0$ and $q_{s, t}+q_{s, t+1}>p_{s, t+1}$ for all $s, t=$

$0, \ldots, n-2$, it follows from the proof of (a) that $\psi_{i}[b]>\psi_{j}[b]$.

$(\Rightarrow)$ (i) First, let us assume that $p_{a, b}+p_{a+1, b} \leq q_{a+1, b}$, for some $a, b=0, \ldots, n-2$.

Pick $i, j \in N$ and $(A, B) \in 3^{N \backslash\{i, j\}}$ such that $|A|=a$ and $|B|=b$. Considerer the identity game $\delta_{(A \cup i, B)}$.

It is clear that $i D j$ and $j \not D i$ in this game. However, $\psi_{i}\left[\delta_{(A \cup i, B)}\right]-\psi_{j}\left[\delta_{(A \cup i, B)}\right]=$ $p_{a, b}+p_{a+1, b}-q_{a+1, b} \leq 0$, a contradiction.

(ii) Now, we assume that $q_{a, b}+q_{a, b+1} \leq p_{a, b+1}$, for some $a, b=0, \ldots, n-2$. Pick $i, j \in N$ and $(A, B) \in 3^{N \backslash\{i, j\}}$ such that $|A|=a$ and $|B|=b$. Considerer the identity game $\delta_{(A, B \cup j)}$.

It is clear that $i D j$ and $j \not D i$ in this game. However, $\psi_{i}\left[\boldsymbol{\delta}_{(A, B \cup j)}\right]-\psi_{j}\left[\boldsymbol{\delta}_{(A, B \cup j)}\right]=$ $q_{a, b}+q_{a, b+1}-p_{a, b+1} \leq 0$, a contradiction.

(iii) Finally, we assume that $p_{a, b+1}+q_{a+1, b}=0$, for some $a, b=0, \ldots, n-2$. Pick $i, j \in N$ and $(A, B) \in 3^{N \backslash\{i, j\}}$ such that $|A|=a$ and $|B|=b$. Considerer the identity game $\delta_{(A \cup i, B \cup j)}$.

Clearly, $i D j$ and $j \not D i$ in this game however, $\psi_{i}\left[\delta_{(A \cup i, B \cup j))}\right]-\psi_{j}\left[\delta_{(A \cup i, B \cup j))}\right]=$ $p_{a, b+1}+q_{a+1, b}=0$, a contradiction.

Remark 3.1 The Shapley and Banzhaf values satisfy the dominance property and the family of $(p, q)$-bisemivalues satisfies dominance property (a).

Now, we introduce regular bisemivalues. 
Definition 3.3 A bisemivalue $\psi$ on $\mathcal{B} \mathcal{G}_{N}$ is regular iff $p_{s, t}>0$ and $q_{s, t}>0$ for all $s, t=0, \ldots, n-1$.

Remark 3.2 (i) The Shapley and the Banzhaf values on bicooperative games are regular bisemivalues. (ii) A $(p, q)$-bisemivalue $\psi^{p q}$ on $\mathcal{B G}_{N}$ is regular iff $p>0, q>0$, and $p+q<1$. (iii) It is easy to see that the regular $(p, q)$-bisemivalues satisfy dominance property (b).

Proposition 3.2 (Indifference property) Let $i, j \in N$ be two distinct players and let $\psi$ be a bisemivalue on $\mathcal{B} \mathcal{G}_{N}$. Then, for every game $b \in \mathcal{B G}_{N}$, iIj implies $\psi_{i}[b]=\psi_{j}[b]$.

Proof Analogously to Proposition 3.1 (a), if $i I j$ we have

$$
\begin{aligned}
\psi_{i}[b]-\psi_{j}[b]= & \sum_{(S, T) \in 3^{N \backslash i, j}}\left[\left(p_{s, t}+p_{s+1, t}-q_{s+1, t}\right)(b(S \cup i, T)-b(S \cup j, T))+\right. \\
& \left(q_{s, t}+q_{s, t+1}-p_{s, t+1}\right)(b(S, T \cup j)-b(S, T \cup i))+ \\
& \left.\left(p_{s, t+1}+q_{s+1, t}\right)(b(S \cup i, T \cup j)-b(S \cup j, T \cup i))\right]=0 .
\end{aligned}
$$

From now on, we will focus on the monotonicity conditions considered in [19] for cooperative games when providing an axiomatic characterization of the Shapley value without using additivity, and extended to semivalues in [18]. Following this work, in the next definition we introduce the monotonicity condition in a similar way as the cooperative case.

Definition 3.4 Let $b_{1}, b_{2} \in \mathcal{B} \mathcal{G}_{N}$ and $i \in N$. We say

$$
b_{1} B b_{2} \text { for i iff }\left\{\begin{array}{l}
b_{1}(S \cup\{i\}, T)-b_{1}(S, T) \geq b_{2}(S \cup\{i\}, T)-b_{2}(S, T), \\
b_{1}(S, T)-b_{1}(S, T \cup\{i\}) \geq b_{2}(S, T)-b_{2}(S, T \cup\{i\}) .
\end{array}\right.
$$

for all $(S, T) \in 3^{N \backslash\{i\}}$. 
That is, $b_{1} B b_{2}$ iff $i$ 's marginal contributions are better (not smaller) in $b_{1}$ than in $b_{2}$.

Relation $B$ allows us to qualitatively compare the positions of a given player $i$ in two games.

Definition 3.5 (a) A value $g$ on $\mathcal{B} \mathcal{G}_{N}$ satisfies the monotonicity property iff $b_{1} B b_{2}$ for $i$, implies $g_{i}\left[b_{1}\right] \geq g_{i}\left[b_{2}\right]$. (b) A value $g$ on $\mathcal{B} \mathcal{G}_{N}$ satisfies the sensitivity property iff $b_{1} B b_{2}$ and $b_{2} B b_{1}$ together for $i$, imply $g_{i}\left[b_{1}\right]>g_{i}\left[b_{2}\right]$.

Proposition 3.3 (Monotonicity and sensitivity properties) Let $\psi$ be a bisemivalue on $\mathcal{B} \mathcal{G}_{N}$ and $b_{1}, b_{2} \in \mathcal{B} \mathcal{G}_{N}$ be distinct games. Then, for each $i \in N$ :

(a) $\psi$ satisfies the monotonicity property.

(b) $b_{1} B b_{2}$ and $b_{2} B b_{1}$ for $i$, implies $\psi_{i}\left[b_{1}\right]=\psi_{i}\left[b_{2}\right]$.

(c) $\psi$ satisfies the sensitivity property iff $\psi$ is regular.

Proof (a) By a mere inspection of

$$
\begin{aligned}
& \psi_{i}\left[b_{1}\right]=\sum_{(S, T) \in 3^{N \backslash i}}\left[p_{s, t}\left(b_{1}(S \cup i, T)-b_{1}(S, T)\right)+q_{s, t}\left(b_{1}(S, T)-b_{1}(S, T \cup i)\right)\right] \\
& \psi_{i}\left[b_{2}\right]=\sum_{(S, T) \in 3^{N \backslash i}}\left[p_{s, t}\left(b_{2}(S \cup i, T)-b_{2}(S, T)\right)+q_{s, t}\left(b_{2}(S, T)-b_{2}(S, T \cup i)\right)\right],
\end{aligned}
$$

it follows that $\psi_{i}\left[b_{1}\right] \geq \psi_{2}\left[b_{1}\right]$, because

$$
\begin{aligned}
& b_{1}(S \cup\{i\}, T)-b_{1}(S, T) \geq b_{2}(S \cup\{i\}, T)-b_{2}(S, T), \\
& b_{1}(S, T)-b_{1}(S, T \cup\{i\}) \geq b_{2}(S, T)-b_{2}(S, T \cup\{i\})
\end{aligned}
$$

and $p_{s, t} \geq 0, q_{s, t} \geq 0$, for all $(S, T) \in 3^{N \backslash\{i\}}$.

(b) It suffices to apply (a) twice.

(c) $(\Leftarrow)$ If $b_{1} B b_{2}$ and $b_{2} B b_{1}$ for $i$, then we have either

$$
\begin{aligned}
& b_{1}(S \cup\{i\}, T)-b_{1}(S, T)>b_{2}(S \cup\{i\}, T)-b_{2}(S, T) \text { or } \\
& b_{1}(S, T)-b_{1}(S, T \cup\{i\})>b_{2}(S, T)-b_{2}(S, T \cup\{i\}),
\end{aligned}
$$


for some $(S, T) \in 3^{N \backslash\{i\}}$. If $\psi$ is regular, $\psi_{i}\left[b_{1}\right]>\psi_{i}\left[b_{2}\right]$ follows from the proof of (a). $(\Rightarrow)$ If $n=1$, the statement holds trivially because any bisemivalue is regular. If $n \geq 2$, let us assume that $\psi$ is not regular. Then, there is either some $p_{s, t}=0$ or $q_{s, t}=0$

(i) Let us first assume that $p_{a, b}=0$, for some $a, b=0, \ldots, n-1$. Pick $i \in N$ and $(A, B) \in 3^{N \backslash\{i\}}$ such that $|A|=a$ and $|B|=b$. Considerer the games $b_{1}=\delta_{(A \cup i, B)}$ and $b_{2}=\frac{1}{2} b_{1}$. Then, $b_{1} B b_{2}$ and $b_{2} B b_{1}$ but, $\psi_{i}\left[b_{1}\right]=p_{a, b}=0=\frac{1}{2} p_{a, b}=\psi_{i}\left[b_{2}\right]$, a contradiction.

(ii) Now, we assume that $q_{a, b}=0$, for some $a, b=0, \ldots, n-1$. Pick $i \in N$ and $(A, B) \in$ $3^{N \backslash\{i\}}$ such that $|A|=a$ and $|B|=b$. Taking $b_{1}=\delta_{(A, B \cup i)}$ and $b_{2}=2 b_{1}$, again it is clear that $b_{1} B b_{2}$ and $b_{2} B b_{1}$ for $\mathrm{i}$ but, $\psi_{i}\left[b_{1}\right]=-q_{a, b}=0=-2 q_{a, b}=\psi_{i}\left[b_{2}\right]$, a contradiction.

\subsection{Nonnull Player, Null Player Exclusion and Balanced Contributions}

\section{Properties}

The first property of this section refers to nonnull players. Usually, if $g$ is a value on $\mathcal{B G}_{N}$, a nonnull player $i \in N$ in a monotonic game $v$ gets a payoff $g_{i}[v]>0$. This property holds for — and in fact characterizes - all regular bisemivalues within the class of bisemivalues.

Proposition 3.4 (Nonnull player property) A bisemivalue on $\mathcal{B G}_{N} \psi$ allocates a positive payoff to every nonnull player in any monotonic game $b \in \mathcal{B} \mathcal{G}_{N}$ if and only if $\psi$ is regular. 
Proof $(\Leftarrow)$ Assume that $\psi$ is regular and let $i \in N$ be a nonnull player in a monotonic game $b \in \mathcal{B} \mathcal{G}_{N}$. Then, by monotonicity, $b(S \cup i, T)-b(S, T) \geq 0$ and $b(S, T)-$ $b(S, T \cup i) \geq 0$ for all $(S, T) \in 3^{N \backslash\{i\}}$ and, either $b(S \cup i, T)-b(S, T)>0$ or $b(S, T)-$ $b(S, T \cup i)>0$, for some $(S, T) \in 3^{N \backslash\{i\}}$, since $i$ is nonnull. Moreover, from regularity, it follows that $p_{s, t}>0$ and $q_{s, t}>0$, for all $s, t=0, \ldots, n-1$. Hence,

$$
\psi_{i}[b]=\sum_{(S, T) \in 3^{N \backslash i}}\left[p_{s, t}(b(S \cup i, T)-b(S, T))+q_{s, t}(b(S, T)-b(S, T \cup i))\right]>0 .
$$

$(\Rightarrow)$ If $n=1, b$ is monotonic and $i$ is no null, the statement holds trivially.

Now, consider $n \geq 2$ and assume that $\psi$ is not regular. Then, there is either some $p_{s, t}=0$ or $q_{s, t}=0$.

(i) If $p_{a, b}=0$ for some $a, b=0, \ldots, n-1$. Pick $i \in N$ and $(A, B) \in 3^{N \backslash\{i\}}$ such that $|A|=a$ and $|B|=b$. Considerer the game

$$
b(S, T)=\left\{\begin{array}{l}
1, \text { if }(S, T) \sqsupseteq(A \cup\{k\}, B) \text { for any } k \in N \backslash(A \cup B), \\
0, \text { otherwise. }
\end{array}\right.
$$

Then, $b$ is monotonic and $i$ is a nonnull player in $b$. However, $\psi_{i}[b]=p_{a, b}=0$.

(ii) If $q_{a, b}=0$, for some $a, b=0, \ldots, n-1$. Pick $i \in N$ and $(A, B) \in 3^{N \backslash\{i\}}$ such that $|A|=a$ and $|B|=b$. Take the game

$$
b(S, T)=\left\{\begin{array}{l}
-1, \text { if }(S, T) \sqsubseteq(A, B \cup\{k\}) \text { for any } k \in N \backslash(A \cup B), \\
0, \quad \text { otherwise. }
\end{array}\right.
$$

Then, $b$ is monotonic and $i$ is a nonnull player in $b$. However, $\psi_{i}[b]=q_{a, b}=0 . \square$

Before studying the following properties, we need the notion of subgame with regard to a nonempty $R \subseteq N$. If $b \in \mathcal{B} \mathcal{G}_{N}$, the game $b_{\mid R} \in \mathcal{G}_{R}$, defined by $b_{\mid R}(S, T)=$ 
$b(S, T)$, for all $(S, T) \subseteq 3^{R}$, is a subgame of $b$. In the particular case where, $T=N \backslash\{i\}$ for some $i \in N$, we will prefer to write $b_{-\{i\}}$ instead of $b_{N \backslash\{i\}}$.

Following [6], analogously to the cooperative case, bisemivalues are also defined on cardinalities rather than on specific player set: that is, two weighting vectors $p_{s, t}$ and $q_{s, t}$ define a bisemivalue $\psi$ on all $N$ such that $n=|N|$. When necessary, we shall write $\psi^{(n)}$ for a bisemivalue on cardinality $n, p_{s, t}^{n}$ and $q_{s, t}^{n}$ for its weighting coefficients. A bisemivalue $\psi^{(n)}$ induces bisemivalues $\psi^{(t)}$ for all cardinalities $t<n$, recurrently defined by the formula given in [6]

$$
\begin{aligned}
& p_{s, t}^{m-1}=p_{s+1, t}^{m}+p_{s, t}^{m}+p_{s, t+1}^{m}, \\
& q_{s, t}^{m-1}=q_{s+1, t}^{m}+q_{s, t}^{m}+q_{s, t+1}^{m}
\end{aligned}
$$

for $0 \leq s, t<m \leq n$.

We will say that $\psi^{(n-1)}, \psi^{(n-2)}, \ldots, \psi^{(1)}$ are the bisemivalues induced by $\psi^{(n)}$.

The following property, introduced for cooperative games in [20], refers to the effect of a null player leaving the game. It is desirable that the payoffs given by a value to the remaining players are not affected by this exclusion. As we will see, this property holds for any bisemivalue.

Definition 3.6 A value $g$ on $\mathcal{B} \mathcal{G}_{N}$ satisfies the null player exclusion property if for all $b \in \mathcal{B} \mathcal{G}_{N} g_{j}[b]=g_{j}\left[b_{-\{i\}}\right]$, for all $i, j \in N$ such that $i$ is a null player in $b$.

Proposition 3.5 (Null player exclusion property) Every bisemivalue $\psi^{(n)}$ on $\mathcal{B G}_{N}$ satisfies the null player exclusion property. 
Proof Following the initial argument of the proof of Proposition 3.1, we obtain

$$
\begin{aligned}
\psi_{j}^{(n)}[b]= & \sum_{(S, T) \in 3^{N \backslash\{j\}}} p_{s, t}^{(n)}[b(S \cup j, T)-b(S, T)]+q_{s, t}^{(n)}[b(S, T)-b(S, T \cup j)]= \\
& \sum_{(S, T) \in 3^{N \backslash\{i, j\}}} p_{s, t}^{(n)}[b(S \cup j, T)-b(S, T)]+q_{s, t}^{(n)}[b(S, T)-b(S, T \cup j)]+ \\
& p_{s+1, t}^{(n)}[b(S \cup i \cup j, T)-b(S \cup i, T)]+q_{s+1, t}^{(n)}[b(S \cup i, T)-b(S \cup i, T \cup j)]+ \\
& p_{s, t+1}^{(n)}[b(S \cup j, T \cup i)-b(S, T \cup i)]+q_{s, t+1}^{(n)}[b(S, T \cup i)-b(S, T \cup j \cup i)] .
\end{aligned}
$$

As $i$ is a null player in $\mathrm{b}$, we can deduce that $b(S \cup i, T)=b(S, T \cup i)=b(S, T)$, $b(S \cup i \cup j, T)=b(S \cup j, T), b(S, T \cup i \cup j)=b(S, T \cup j), b(S \cup j, T \cup i)=b(S \cup j, T)$ and $b(S \cup i, T \cup j)=b(S, T \cup j)$.

Thus, using (4)

$$
\begin{aligned}
\psi_{j}^{(n)}[b]= & \sum_{(S, T) \in 3^{N \backslash\{i, j\}}}\left(p_{s+1, t}^{n}+p_{s, t}^{n}+p_{s, t+1}^{n}\right)[b(S \cup j, T)-b(S, T)]+ \\
& \left(q_{s+1, t}^{n}+q_{s, t}^{n}+q_{s, t+1}^{n}\right)[b(S, T)-b(S, T \cup j)] \\
= & \left.\left.\sum_{(S, T) \in 3^{N \backslash\{i, j\}}} p_{s, t}^{(n-1)}\left[b_{-\{i\}}\right](S \cup j, T)-b_{-\{i\}}\right](S, T)\right]+ \\
& \left.\left.q_{s, t}^{(n-1)}\left[b_{-\{i\}}\right](S, T)-b_{-\{i\}}\right](S, T \cup j)\right] \\
= & \psi_{j}^{(n-1)}\left[b_{-\{i\}}\right] .
\end{aligned}
$$

The following property, originally introduced in [21] for cooperative games, refers to the effect of excluding a player on the payoff to any other player.

Definition 3.7 A value $g$ on $\mathcal{B} \mathcal{G}_{N}$ satisfies the property of balanced contributions if for all $b \in \mathcal{B} \mathcal{G}_{N}$ and all $i, j \in N, g_{i}[b]-g_{i}\left[b_{-\{j\}}\right]=g_{j}[b]-g_{j}\left[b_{-\{i\}}\right]$.

In the above equation, the left-hand side represents $j^{\prime} s$ contributions to $i^{\prime} s$ payoff, while the right-hand side represents $j^{\prime} s$ contributions to $i^{\prime} s$ payoff. This property 
states that these contributions are balanced between any two players. In the next proposition, we characterize the class of solutions satisfying the balanced contributions property within the class of bisemivalues.

Proposition 3.6 (Balanced contributions property) Let $\psi^{(n)}$ be a bisemivalue on $\mathcal{B G}_{N}$.

Then,

$\psi^{(n)}$ satisfies the balanced contributions property iff $p_{s, t+1}^{n}+q_{s+1, t}^{n}=0, \quad$ for all $s, t=0, .1, \ldots, n-2$.

Proof $(\Leftarrow)$ Let $\psi^{(n)}$ be a bisemivalue on cardinality $n$. Starting at Eq. (3) and using Eq. (4), after some calculus we have

$\psi_{i}^{(n)}[b]-\psi_{i}^{(n-1)}\left[b_{-\{j\}}\right]=$

$$
\begin{aligned}
\sum_{(S, T) \in 3^{N \backslash i, j}} & p_{s+1, t}^{n}[b(S \cup j \cup i, T)-b(S \cup j, T)-b(S \cup i, T)+b(S, T)]+ \\
& q_{s+1, t}^{n}[b(S \cup j, T)-b(S \cup j, T \cup i)-b(S, T)+b(S, T \cup i)]+ \\
& p_{s, t+1}^{n}[b(S \cup i, T \cup j)-b(S, T \cup j)-b(S \cup i, T)+b(S, T)]+ \\
& \left.q_{s, t+1}^{n}[b(S, T \cup j)-b(S, T \cup i \cup j)-b(S, T)+b(S, T \cup i)]\right] .
\end{aligned}
$$

Now, by comparing it with the analogous expression for $\psi_{j}^{(n)}[b]-\psi_{j}^{(n-1)}\left[b_{-\{i\}}\right]$ and taking into account that $p_{s, t+1}^{n}=q_{s+1, t}^{n}=0$, it follows that

$$
\begin{aligned}
& {\left[\psi_{i}^{(n)}[b]-\psi_{i}^{(n-1)}\left[b_{-\{j\}}\right]\right]-\left[\psi_{j}^{(n)}[b]-\psi_{j}^{(n-1)}\left[b_{-\{i\}}\right]\right]=\sum_{(S, T) \in 3^{N \backslash\{i, j\}}}\left(p_{s, t+1}^{n}+q_{s+1, t}^{n}\right)} \\
& {[b(S \cup j, T)-b(S \cup i, T)+b(S \cup i, T \cup j)-b(S, T \cup j)-b(S \cup j, T \cup i)+b(S, T \cup i)]} \\
& =0
\end{aligned}
$$

Then, $\quad \psi_{i}^{(n)}[b]-\psi_{i}^{(n-1)}\left[b_{-\{j\}}\right]=\psi_{j}^{(n)}[b]-\psi_{j}^{(n-1)}\left[b_{-\{i\}}\right]$ 
$(\Rightarrow)$ Assume that $p_{a, b+1}^{n}+q_{a+1, b}^{n} \neq 0$, for some $a, b=0, \ldots, n-2$. Pick $i, j \in N$ and $(A, B) \in 3^{N \backslash\{i, j\}}$ such that $|A|=a$ and $|B|=b$.

If we considerer the identity game $\delta_{(A \cup i, B \cup j)}$, we have

$$
\begin{aligned}
& {\left[\psi_{i}^{(n)}\left[\boldsymbol{\delta}_{(A \cup i, B \cup j)}\right]-\psi_{i}^{(n-1)}\left[\boldsymbol{\delta}_{(A \cup i, B \cup j)-\{j\}}\right]\right]-\left[\psi_{j}^{(n)}\left[\boldsymbol{\delta}_{(A \cup i, B \cup j)}\right]-\psi_{j}^{(n-1)}\left[\boldsymbol{\delta}_{(A \cup i, B \cup j)-\{i\}}\right]\right]=} \\
& p_{a, b+1}^{n}+q_{a+1, b}^{n} \neq 0, \text { a contradiction. }
\end{aligned}
$$

\subsection{Block Property}

To study the following property we need to define formally what is meant by two players, $i$ and $j$ in a bicooperative game forming a block and operating as a single player. Clearly, this gives rise to a new bicooperative game whose set of players is obtained from the initial set of players by removing both $i$ and $j$ and introducing a new player representing the block. From now on, we will denote this new player by $i \& j$ and $b_{i \& j}$ the corresponding bicooperative game.

Definition 3.8 Let $b \in \mathcal{B} \mathcal{G}_{N}$ and let $i$ and $j$ be two distinct players in $N$. Taking $i \& j$ to be a new entity, not belonging to $N$, the game $b_{i \& j}$ played in $N^{\prime}=N \backslash\{i, j\} \cup\{i \& j\}$ is defined by

$$
b_{i \& j}(S, T)= \begin{cases}b(S \backslash\{i \& j\} \cup\{i\} \cup\{j\}, T), & \text { if } i \& j \in S, \\ b(S, T \backslash\{i \& j\} \cup\{i\} \cup\{j\}), & \text { if } i \& j \in T, \\ b(S, T), & \text { otherwise. }\end{cases}
$$

Definition 3.9 A value $g$ on $\mathcal{B} \mathcal{G}_{N}$ satisfies the block property if for every monotonic game $b \in \mathcal{B} \mathcal{G}_{N}$ and for all $i, j \in N$ such that $j$ is not a null player then, $g_{i \& j}\left[b_{i \& j}\right]>$ $g_{i}[b]$. 
Proposition 3.7 (Block property) Let $\psi^{(n)}$ be a bisemivalue on $\mathcal{B} \mathcal{G}_{N}$. Then, $\psi^{(n)}$ satisfies the block property iff $p_{s+1, t}^{n}-q_{s+1, t}^{n}>0, \quad q_{s, t+1}^{n}-p_{s, t+1}^{n}>0, p_{s, t}^{n}+p_{s, t+1}^{n}>0$ and $q_{s, t}^{n}+q_{s+1, t}^{n}>0$, for all $s, t=0, .1, \ldots, n-2$.

Proof $(\Leftarrow)$ Let $\psi^{(n)}$ be a bisemivalue on cardinality $n$. Starting at Eq. (3) and using Eq. (4), after some calculus, we have

$$
\begin{aligned}
\psi_{i \& j}^{(n-1}\left[b_{i \& j}\right]-\psi_{i}^{(n)}[b] & =\sum_{(S, T) \in 3^{N \backslash\{i, j\}}} p_{s, t}^{n}[b(S \cup i \cup j, T)-b(S \cup i, T)]+ \\
& \left(p_{s+1, t}^{n}-q_{S+1, t}^{n}\right)[b(S \cup j, T)-b(S, T)]+q_{S+1, t}^{n}[b(S \cup j, T \cup i)-b(S, T \cup i \cup j)]+ \\
& \left(q_{s, t+1}^{n}-p_{s, t+1}^{n}\right)[b(S, T)-b(S, T \cup j)]+p_{s, t+1}^{n}[b(S \cup i \cup j, T)- \\
& b(S \cup i, T \cup j)]+q_{s, t}^{n}[b(S, T \cup i)-b(S, T \cup i \cup j)]
\end{aligned}
$$

By monotonicity,

$$
\begin{aligned}
& b(S \cup i \cup j, T)-b(S \cup i, T) \geq 0, \quad b(S \cup j, T)-b(S, T) \geq 0, \\
& b(S \cup j, T \cup i)-b(S, T \cup i \cup j) \geq 0, \quad b(S, T)-b(S, T \cup j) \geq 0, \\
& b(S \cup i \cup j, T)-b(S \cup i, T \cup j) \geq 0 \text { and } b(S, T \cup i)-b(S, T \cup i \cup j) \geq 0,
\end{aligned}
$$

for all $(S, T) \in 3^{N \backslash\{i, j\}}$ and either

(i) $b(S \cup i \cup j, T)-b(S \cup i, T)>0$ or $(i i) b(S \cup j, T)-b(S, T)>0$ or (iii) $b(S, T)-b(S, T \cup j)>0$ or $(i v) b(S, T \cup i)-b(S, T \cup i \cup j)>0$, for some $(S, T) \in 3^{N \backslash\{i, j\}}$, since $j$ is nonnull.

If (i), by monotonicity, $b(S \cup i \cup j, T)-b(S \cup i, T \cup j)>0$ and if $p_{s, t}=0$ then, $p_{s, t+1}>0$.

If either (ii) or (iii), the property holds taking into account that $p_{s+1, t}^{n}-q_{s+1, t}^{n}>0$ and $q_{s, t+1}^{n}-p_{s, t+1}^{n}>0$. 
If (iv), by monotonicity, $b(S \cup j, T \cup i)-b(S, T \cup i \cup j)>0$ and if $q_{s, t}=0$ then, $q_{s+1, t}>0$. In all cases we get $\psi_{i \& j}^{(n-1)}\left[b_{i \& j}\right]-\psi_{i}^{(n)}[b]>0$.

$(\Rightarrow)$

(i) Assume that $p_{a+1, b}^{n}-q_{a+1, b}^{n} \leq 0$, for some $a, b=0, \ldots, n-2$. Pick $i, j \in N$ and $(A, B) \in 3^{N \backslash\{i, j\}}$ such that $|A|=a$ and $|B|=b$.

We considerer the game

$$
b(S, T)= \begin{cases}1, \text { if either }(S, T) \sqsupseteq(A \cup\{k\}, B) \text { or }(S, T) \sqsupseteq(A \cup\{k\}, B \cup\{j\}) & \text { for any } k \in N \backslash(A \cup B), \\ 0, \text { otherwise. } & \end{cases}
$$

$b$ is monotonic and $j$ is a nonnull player in $b$.

However, $\psi_{i \& j}^{(n-1)}\left[b_{i \& j}\right]-\psi_{i}^{(n)}[b]=p_{a+1, b}^{n}-q_{a+1, b}^{n} \leq 0$, a contradiction.

(ii) Assume that $q_{a, b+1}^{n}-p_{a, b+1}^{n} \leq 0$, for some $a, b=0, \ldots, n-2$. Pick $i, j \in N$ and $(A, B) \in 3^{N \backslash\{i, j\}}$ such that $|A|=a$ and $|B|=b$. We consider the game

$$
b(S, T)=\left\{\begin{array}{l}
1, \text { if either }(S, T) \sqsupseteq(A, B) \text { or }(S, T) \sqsupseteq(A \cup\{i\}, B \cup\{j\}), \\
0, \text { otherwise. }
\end{array}\right.
$$

$b$ is monotonic and $j$ is a nonnull player in $b$.

However, $\psi_{i \& j}^{(n-1)}\left[b_{i \& j}\right]-\psi_{i}^{(n)}[b]=q_{a, b+1}^{n}-p_{a, b+1}^{n} \leq 0$, a contradiction.

(iii) Assume that $p_{a, b}^{n}+p_{a, b+1}^{n}=0$, for some $a, b=0, \ldots, n-2$. Pick $i, j \in N$ and $(A, B) \in 3^{N \backslash\{i, j\}}$ such that $|A|=a$ and $|B|=b$. We consider the game

$$
b(S, T)=\left\{\begin{array}{l}
1, \text { if }(S, T) \sqsupseteq(A \cup\{k\} \cup\{l\}, B) \text { for any } k, l \in N \backslash(A \cup B), \\
0, \text { otherwise. }
\end{array}\right.
$$

$b$ is monotonic and $j$ is a nonnull player in $b$.

However, $\psi_{i \& j}^{(n-1)}\left[b_{i \& j}\right]-\psi_{i}^{(n)}[b]=p_{a, b}^{n}+p_{a, b+1}^{n}=0$, a contradiction. 
(iv) Assume that $q_{a, b}^{n}+q_{a+1, b}^{n}=0$, for some $a, b=0, \ldots, n-2$. Pick $i, j \in N$ and $(A, B) \in 3^{N \backslash\{i, j\}}$ such that $|A|=a$ and $|B|=b$. We considerer the game

$$
b(S, T)=\left\{\begin{array}{l}
1, \text { if }(S, T) \sqsupseteq(A, B \cup\{k\}) \text { for any } k \in N \backslash(A \cup B), \\
0, \text { otherwise. }
\end{array}\right.
$$

$b$ is monotonic and $j$ is a nonnull player in $b$.

However, $\psi_{i \& j}^{(n-1)}\left[b_{i \& j}\right]-\psi_{i}^{(n)}[b]=q_{a, b}^{n}+q_{a+1, b}^{n}=0$, a contradiction.

Notice that the block $i \& j$ can be regarded as a result of a voluntary merger between players $i$ and $j$. But it can also be regarded as a result of a takeover, in which $i$, having annexed $j^{\prime} s$ player rights, now trades under the new name $i \& j$. The assumption that player $i$ must gain power by absorbing in this way player $j$, who is not a null player, seems to be intuitively compelling. A voluntary merger will take place only if a result of it, both parties are at least as well as they were before. A takeover, however, need not be beneficial to both parties, but only to the one instigating it.

\section{A Computational Procedure to Calculate Bisemivalues}

As it is well known, both the Shapley and Banzhaf values of any cooperative game $v$ can be easily obtained from its multilinear extension $[13,14]$. This latter procedure extends well to any $p$-binomial semivalue (see, e.g., [22,23]). Also, in [9], semivalues on cooperative games are computed by means of the MLE of the game.

Following this idea, the multilinear extension of a bicooperative game is introduced in [6] in a similar way as in the cooperative case [14] and it allows us to compute $(p, q)$ - bisemivalues in general, and the Banzhaf bisemivalue in particular. 
In this section, we go further and present a method to compute any bisemivalue by means of the multilinear extension of the game.

Following [6], each $(S, T) \in 3^{N}$ is identified by a vector $(X, Y)$ of $\mathbb{R}^{2 n}$ such that $X=\left(x_{1}, \ldots, x_{n}\right), Y=\left(y_{1}, \ldots, y_{n}\right)$, and

$$
x_{i}=\left\{\begin{array}{ll}
1, & \text { if } i \in S, \\
0, & \text { otherwise }
\end{array} \quad \text { and } \quad y_{i}= \begin{cases}1, & \text { if } i \in T, \\
0, & \text { otherwise }\end{cases}\right.
$$

For instance, if $N=\{1,2,3\}$ the coalitions $(\{1,3\},\{2\})$ and $(\{1,2\}, \emptyset)$ are identified by $(X, Y)=(1,0,1,0,1,0)$ and $(X, Y)=(1,1,0,0,0,0)$, respectively.

Definition 4.1 (Domènech et al, 2018) The multilinear extension of a game $b \in \mathcal{B} \mathcal{G}_{N}$ is the real-valued function defined on $\mathbb{R}^{2 n}$ by

$$
f(X, Y)=\sum_{(S, T) \in 3^{N}}\left[\prod_{i \in S} x_{i} \prod_{j \in T} y_{j} \prod_{k \in N \backslash(S \cup T)}\left(1-x_{k}-y_{k}\right)\right] b(S, T) .
$$

Lemma 4.1 Let $(A, B) \in 3^{N}$ such that $(A, B) \neq(\emptyset, \emptyset)$. Then, the MLE of the identity game $\delta_{(A, B)}$ is

$$
f(X, Y)=\prod_{i \in A} x_{i} \prod_{j \in B} y_{j} \prod_{k \in N \backslash(A \cup B)}\left(1-x_{k}-y_{k}\right)
$$

Lemma 4.2 Let $\psi$ be a bisemivalue on $\mathcal{B} \mathcal{G}_{N}$. The allocations to a player $i \in N$ in the identity game $\delta_{(A, B)}$ are given by

$$
\psi_{i}\left[\boldsymbol{\delta}_{(A, B)}\right]= \begin{cases}p_{a-1, b}, & \text { if } i \in A, \\ -q_{a, b-1}, & \text { if } i \in B, \\ q_{a, b}-p_{a, b}, & \text { if } i \in N \backslash(A \cup B) .\end{cases}
$$

Proof It easily follows by applying the definition of bisemivalue given in Theorem 2.1 to the identity game $\delta_{(A, B)}$. 
In the next theorem, we present a method to compute any bisemivalue by means of the multilinear extension of the game.

Theorem 4.1 Let $b \in \mathcal{B} \mathcal{G}_{N}$ and $\psi$ be a bisemivalue on $\mathcal{B G}_{N}$ with weighting coefficients $p_{s, t}$ and $q_{s, t}, s, t=0,1, \ldots, n-1$. Then, the following steps lead to the bisemivalue value of any player $i \in N$.

1. Obtain the multilinear extension $f(X, Y)$ of game $b$.

2. For each $i \in N$, obtain a new multilinear function called $f^{i}$ multiplying each prod-

uct

$$
\prod_{j \in S} x_{j} \prod_{k \in T} y_{k} \prod_{l \in N \backslash(S \cup T)}\left(1-x_{l}-y_{l}\right)
$$

by $p_{s-1, t}$ if $i \in S$; by $-q_{s, t-1}$ if $i \in T$ and by $\left(q_{s, t}-p_{s, t}\right)$ if $i \in N \backslash(S \cup T)$.

3. Avaluate $f^{i}$ at point $\left(\mathbf{1}_{S}, \mathbf{1}_{T}\right)$, where

$$
\boldsymbol{1}_{S}=\left\{\begin{array}{ll}
1, & \text { if } j \in S, \\
0, & \text { if } j \in N \backslash(S \cup T) .
\end{array} \text { and } \quad \mathbf{1}_{T}=\left\{\begin{array}{l}
1, \quad \text { if } j \in T, \\
0, \quad \text { if } j \in N \backslash(S \cup T),
\end{array}\right.\right.
$$

for each $j \in N$ and $\psi_{i}[b]=f^{i}\left(\boldsymbol{1}_{S}, \boldsymbol{1}_{T}\right)$,

Proof Step 1 shows the multilinear extension of $b$ as a linear combination of multilinear extensions of identity games. Step 2 weights each identity game according to Lemma 4.2 in order to obtain $\psi_{i}[b]$ in step 3.

\section{An Example}

In this section, we present an example of bicooperative game (for more specific examples about bicooperative games, we refer the reader to $[6,24])$. The allocations 
obtained by the players will be analyzed by using bisemivalues and we will compute them by using the MLE technique given in Theorem 4.1.

The Navigator company has 3 investors. The investments of each one of them are 2, 4 and 6 million euros respectively. The company is studying a new project where, for every million invested, 1.5 million will be received. Now, Navigator company needs to know investors' intentions of investing in this project. If $N=\{1,2,3\}$ is the set of investors, we can define the bicooperative game $b(S, T)$ as the company profit when players in $S$ agree to invest their investment in the project and players in $T$ do not agree to the project and remove their investment to the company. Finally, abstention means that the investor does not invest in the project, but does not withdraw his investment in the company. In this situation, $b$ is the bicooperative game defined by

$$
\begin{array}{llll}
b(\{1,2,3\}, \emptyset)=6, & b(\emptyset, \emptyset)=0, & b(\emptyset,\{1,2,3\})=-12, & b(\emptyset,\{1\})=-2, \\
b(\{1,3\}, \emptyset)=4, & b(\{2,3\}, \emptyset)=5, & b(\{1,2\}, \emptyset)=3, & b(\emptyset,\{2\})=-4, \\
b(\{1,3\},\{2\})=0, & b(\{2,3\},\{1\})=3, & b(\{1,2\},\{3\})=-3, & b(\emptyset,\{3\})=-6, \\
b(\{3\}, \emptyset)=3, & b(\{2\}, \emptyset)=2, & b(\{1\}, \emptyset)=1, & b(\emptyset,\{2,3\})=-10, \\
b(\{3\},\{1\})=1, & b(\{3\},\{2\})=-1, & b(\{2\},\{1\})=0, & b(\emptyset,\{1,3\})=-8, \\
b(\{2\},\{3\})=-4, & b(\{1\},\{2\})=-3, & b(\{1\},\{3\})=-5, & b(\emptyset,\{1,2\})=-6, \\
b(\{3\},\{1,2\})=-3, & b(\{2\},\{1,3\})=-6, & b(\{1\},\{2,3\})=-9 . &
\end{array}
$$

From Definition 4.1 the MLE of $b$ is

$$
\begin{aligned}
f(X, Y)= & 6 x_{1} x_{2} x_{3}+4 x_{1} x_{3}\left(1-x_{2}-y_{2}\right)+3 x_{3}\left(1-x_{1}-y_{1}\right)\left(1-x_{2}-y_{2}\right)+x_{3} y_{1}\left(1-x_{2}-y_{2}\right)- \\
& 4 x_{2} y_{3}\left(1-x_{1}-y_{1}\right)-3 x_{3} y_{1} y_{2}-2 y_{1}\left(1-x_{2}-y_{2}\right)\left(1-x_{3}-y_{3}\right)-10 y_{2} y_{3}\left(1-x_{1}-y_{1}\right)+ \\
& 5 x_{2} x_{3}\left(1-x_{1}-y_{1}\right)+3 x_{2} x_{3} y_{1}+2 x_{2}\left(1-x_{1}-y_{1}\right)\left(1-x_{3}-y_{3}\right)-x_{3} y_{2}\left(1-x_{1}-y_{1}\right)- \\
& 3 x_{1} y_{2}\left(1-x_{3}-y_{3}\right)-6 x_{2} y_{1} y_{3}-4 y_{2}\left(1-x_{1}-y_{1}\right)\left(1-x_{3}-y_{3}\right)-8 y_{1} y_{3}\left(1-x_{2}-y_{2}\right)- \\
& 12 y_{1} y_{2} y_{3}+3 x_{1} x_{2}\left(1-x_{3}-y_{3}\right)-3 x_{1} x_{2} y_{3}+x_{1}\left(1-x_{2}-y_{2}\right)\left(1-x_{3}-y_{3}\right)- \\
& 5 x_{1} y_{3}\left(1-x_{2}-y_{2}\right)-9 x_{1} y_{2} y_{3}-6 y_{3}\left(1-x_{1}-y_{1}\right)\left(1-x_{2}-y_{2}\right)-6 y_{1} y_{2}\left(1-x_{3}-y_{3}\right)
\end{aligned}
$$


We will compute $\psi[b]$, where $\psi$ is a bisemivalue on $\mathcal{B} \mathcal{G}_{N}$ with weighting coefficients $p_{s, t}$ and $q_{s, t}, s, t=0,1,2$, by using the computational method given in Section 4. Step

2 in Theorem 4.1 gives a new MLE $f^{i}$, for each $i=1,2,3$. For player 1 ,

$f^{1}(X, Y)=6 p_{2,0} x_{1} x_{2} x_{3}+4 p_{1,0} x_{1} x_{3}\left(1-x_{2}-y_{2}\right)+3\left(q_{1,0}-p_{1,0}\right) x_{3}\left(1-x_{1}-y_{1}\right)\left(1-x_{2}-y_{2}\right)-$

$$
\begin{aligned}
& q_{1,0} x_{3} y_{1}\left(1-x_{2}-y_{2}\right)-4\left(q_{1,1}-p_{1,1}\right) x_{2} y_{3}\left(1-x_{1}-y_{1}\right)+3 q_{1,1} x_{3} y_{1} y_{2}+ \\
& 2 q_{0,0} y_{1}\left(1-x_{2}-y_{2}\right)\left(1-x_{3}-y_{3}\right)-10\left(q_{0,2}-p_{0,2}\right) y_{2} y_{3}\left(1-x_{1}-y_{1}\right)+ \\
& 5\left(q_{2,0}-p_{2,0}\right) x_{2} x_{3}\left(1-x_{1}-y_{1}\right)-3 q_{2,0} x_{2} x_{3} y_{1}+2\left(q_{1,0}-p_{1,0}\right) x_{2}\left(1-x_{1}-y_{1}\right)\left(1-x_{3}-y_{3}\right)- \\
& \left(q_{1,1}-p_{1,1}\right) x_{3} y_{2}\left(1-x_{1}-y_{1}\right)-3 p_{0,1} x_{1} y_{2}\left(1-x_{3}-y_{3}\right)+6 q_{1,1} x_{2} y_{1} y_{3}- \\
& 4\left(q_{0,1}-p_{0,1}\right) y_{2}\left(1-x_{1}-y_{1}\right)\left(1-x_{3}-y_{3}\right)+8 q_{0,1} y_{1} y_{3}\left(1-x_{2}-y_{2}\right)+12 q_{0,2} y_{1} y_{2} y_{3}+ \\
& 3 p_{1,0} x_{1} x_{2}\left(1-x_{3}-y_{3}\right)-3 p_{1,1} x_{1} x_{2} y_{3}+p_{0,0} x_{1}\left(1-x_{2}-y_{2}\right)\left(1-x_{3}-y_{3}\right)-5 p_{0,1} x_{1} y_{3}\left(1-x_{2}-y_{2}\right)- \\
& 9 p_{0,2} x_{1} y_{2} y_{3}-6\left(q_{0,1}-p_{0,1}\right) y_{3}\left(1-x_{1}-y_{1}\right)\left(1-x_{2}-y_{2}\right)+6 q_{0,1} y_{1} y_{2}\left(1-x_{3}-y_{3}\right)
\end{aligned}
$$

Step 3 yields

$$
\psi_{1}[b]=p_{0,0}+2 p_{1,0}+2 p_{0,1}+p_{2,0}+2 p_{1,1}+p_{0,2}+2 q_{0,0}+4 q_{1,0}+4 q_{0,1}+2 q_{2,0}+4 q_{1,1}+2 q_{0,2}
$$

Analogously, for players 2 and 3:

$$
\begin{aligned}
& \psi_{2}[b]=2 p_{0,0}+4 p_{1,0}+4 p_{0,1}+2 p_{2,0}+4 p_{1,1}+2 p_{0,2}+4 q_{0,0}+8 q_{1,0}+8 q_{0,1}+4 q_{2,0}+8 q_{1,1}+4 q_{0,2}, \\
& \psi_{3}[b]=3 p_{0,0}+6 p_{1,0}+6 p_{0,1}+3 p_{2,0}+6 p_{1,1}+3 p_{0,2}+6 q_{0,0}+12 q_{1,0}+12 q_{0,1}+6 q_{2,0}+12 q_{1,1}+6 q_{0,2} .
\end{aligned}
$$

Particularly, for Shapley bisemivalue $\varphi$, whose weighting coefficients are

$p_{s, t}=\frac{(3+s-t) !(3+t-s-1) !}{6 !} 2^{3-s-t}$ and $q_{s, t}=\frac{(3+t-s) !(3+s-t-1) !}{6 !} 2^{3-s-t}$,

for $s=0,1,2$, we get: $\varphi_{1}[b]=3, \varphi_{2}[b]=6, \varphi_{3}[b]=9$.

Notice that $\sum_{i \in N} \varphi_{i}[b]=b(N, \emptyset)-b(\emptyset, N)=18$.

\section{Conclusions}

We investigate the conditions for the coefficients of the bisemivalues necessary and / or sufficient in order to satisfy some properties. Most of these properties were studied 
in the context of cooperative games: in [5], for several power indices; in [18], for semivalues; in [7], for semiindices (semivalues for simple games) and in [8], for probabilistic values in general and multinomial (probabilistic) values in particular. The study of properties is a useful tool to know better the available solutions for a model. We can use it in order to identify the most appropiate solution for a particular problem by choosing that one whose supporting properties are more adequate for the problem. In this work, we also characterize different families of bisemivalues by means of algebraic expressions of the coefficients: (i) the class of bisemivalues that satisfies the dominance property; (ii) the regular bisemivalues, as those that satisfy the sensitive property, (iii) the regular bisemivalues, as those that allocate positive payoffs to every nonnull player in a monotonic game, (iv) the class of bisemivalues that satisfies the property of balanced contributions and (v) the class of bisemivalues satisfying the block property.

Acknowledgements This research project was partially supported by funds from the Spanish Ministry of Economy and Competitiveness (MINECO) and from the European Union (FEDER funds) under grant MTM2015-66818-P (MINECO/FEDER) and Grant SGR 2017-758 of the Catalonia Government (Generalitat de Catalunya).

The authors wish to thank the anonymous reviewers for their helpful suggestions which have been incorporated to the text.

\section{References}

1. Bilbao, J.M.: Cooperation games on combinatorial structures. Kluwer Ac. Publishers, Boston (2000).

2. Bilbao, J.M., Fernández, J.R., Jimenez, N. and López, JJ.: The Shapley value for bicooperative games. Annals of Operations Research 158, 99-115 (2000)

3. Bilbao, J.M., Fernández, J.R., Jimenez, N. and López, JJ.: Biprobabilistic values for bicooperative games. Discrete Applied Mathematics 156, 2698-2711 (2008). 
4. Bilbao, J.M., Fernández, J.R., Jimenez, N. and López, JJ.: The Banzhaf power index for ternary bicooperative games. Discrete Applied Mathematics 158, 967-980 (2010)

5. Felsenthal, D. and Machover, M.: Ternary voting games. International Journal of Game Theory 26, $335-351(1997)$

6. Domènech M., Giménez, J.M and Puente, M.A.: Bisemivalues for bicooperative games. Optimization 67:6, 907-919 (2018)

7. Carreras, F., Freixas, J. and Puente, M. A.: Semivalues as power indices. European Journal of Operational Research 149, 676-687 (2003)

8. Domènech, M., Giménez, J.M. and Puente, M.A.: Some properties for probabilistic and multinomial (probabilistic) values on cooperative games. Optimization 65, 1377-1395 (2016)

9. Carreras, F., Giménez, J.M.: Power and potential maps induced by any semivalue: Some algrebraic properties and computation by multilinear extension. European Journal of Operational Research 211, $148-159(2011)$

10. Weber, R.J. ]: Probabilistic values for games. In: The Shapley Value: Essays in Honor of Lloyd S. Shapley (A.E. Roth, ed.), Cambridge University Press, 101-119 (1988)

11. Dubey, P., Neyman, A. and Weber, R.J.: Value theory without efficiency. Mathematics of Operations Research 6, 122-128 (1981)

12. Shapley, L.S.: A value for n-person games. In: Contributions to the Theory of Games II (H.W. Kuhn and A.W. Tucker, eds.), Princeton University Press, 307-31 (1953)

13. Owen, G.: Multilinear extensions and the Banzhaf value. Naval Research Logistics Quarterly 22, $741-750(1975)$

14. Owen, G.: Multilinear extensions of games. Management Science 18, 64-79 (1972)

15. Grabisch, M. and Labreuche, Ch.; Bi-capacities-I: Definition, Möbius transform and interaction. Fuzzy Sets and Systems 151, 211-236 (2005)

16. Puente, M.A. : Aportaciones a la representabilidad de juegos simples y al cálculo de soluciones de esta clase de juegos (in Spanish). Ph.D. Thesis. Technical University of Catalonia, Spain, (2000)

17. Isbell, J.R.: A class of simple games. Duke Mathematics Journal 25, 423-439 (1958)

18. Carreras, F. and Freixas, J.: Some theoretical reasons for using (regular) semivalues. In: Logic, Game Theory and Social Choice (H. de Swart, ed.), Tilburg University Press, 140-154 (1999) 
19. Young, H.P. : Monotonic solutions of cooperative games. International Journal of Game Theory 14, $65-72(1985)$

20. Derks, J.J.M. and Haller, H.H.: Null players out? Linear values for games with variables supports. International Game Theory Review 1, 301-314 (1999)

21. Myerson, R.: Conference structures and fair allocation rules. International Journal of Game Theory 9, 169-182 (1980)

22. Freixas, J. and Puente, M.A. : Reliability importance measures of the components in a system based on semivalues and probabilistic values. Annals of Operations Research 109, 331-342 (2002)

23. Amer, R. and Giménez, J.M.: Modification of semivalues for games with coalition structures. Theory and Decision 54, 185-205 (2003)

24. Grabisch, M. and Labreuche, Ch.: Axiomatization of the Shapley value and power index for bicooperative games. Cahiers de la Maison des Sciences Economiques 2006.23 - ISSN 1624-0340. $<$ halshs $-00113340>(2006)$ 